KYUNGPOOK Math. J. 51(2011), 241-250

http://dx.doi.org/10.5666/KMJ.2011.51.3.241

\title{
Coefficient Inequalities for Certain Subclasses of Analytic Functions Defined by Using a General Derivative Operator
}

SERAP Bulut

Kocaeli University, Civil Aviation College, Arslanbey Campus, 41285, IzmitKocaeli, Turkey

e-mail : serap.bulut@kocaeli.edu.tr

ABstract. In this paper, we define new classes of analytic functions using a general derivative operator which is a unification of the Sălăgean derivative operator, the OwaSrivastava fractional calculus operator and the Al-Oboudi operator, and discuss some coefficient inequalities for functions belong to this classes.

\section{Introduction}

Let $\mathcal{A}$ denote the class of all functions of the form

$$
f(z)=z+\sum_{k=2}^{\infty} a_{k} z^{k}
$$

which are analytic in the open unit disk $\mathbb{U}=\{z \in \mathbb{C}:|z|<1\}$, and $\mathcal{S}=$ $\{f \in \mathcal{A}: f$ is univalent in $\mathbb{U}\}$.

The following definition of fractional derivative by Owa [8] (also by Srivastava and Owa [18]) will be required in our investigation.

The fractional derivative of order $\gamma$ is defined, for a function $f$, by

$$
D_{z}^{\gamma} f(z)=\frac{1}{\Gamma(1-\gamma)} \frac{d}{d z} \int_{0}^{z} \frac{f(\xi)}{(z-\xi)^{\gamma}} d \xi \quad(0 \leq \gamma<1),
$$

where the function $f$ is analytic in a simply connected region of the complex $z$-plane containing the origin, and the multiplicity of $(z-\xi)^{-\gamma}$ is removed by requiring $\log (z-\xi)$ to be real when $z-\xi>0$.

It readily follows from $(1.2)$ that

$$
D_{z}^{\gamma} z^{k}=\frac{\Gamma(k+1)}{\Gamma(k+1-\gamma)} z^{k-\gamma} \quad(0 \leq \gamma<1, k \in \mathbb{N}=\{1,2, \ldots\}) .
$$

Received December 2, 2009; revised November 9, 2010; accepted September 14, 2011. 2010 Mathematics Subject Classification: 30C45, 30C50.

Key words and phrases: Analytic function, Sălăgean derivative operator, Owa-Srivastava fractional calculus operator, Al-Oboudi operator. 
Using $D_{z}^{\gamma} f$, Owa and Srivastava [10] introduced the operator $\Omega^{\gamma}: \mathcal{A} \rightarrow \mathcal{A}$, which is known as an extension of fractional derivative and fractional integral, as follows:

$$
\begin{aligned}
\Omega^{\gamma} f(z) & =\Gamma(2-\gamma) z^{\gamma} D_{z}^{\gamma} f(z), \quad \gamma \neq 2,3,4, \ldots \\
& =z+\sum_{k=2}^{\infty} \frac{\Gamma(k+1) \Gamma(2-\gamma)}{\Gamma(k+1-\gamma)} a_{k} z^{k} .
\end{aligned}
$$

Note that

$$
\Omega^{0} f(z)=f(z) .
$$

In [3], Al-Oboudi and Al-Amoudi defined the linear multiplier fractional differential operator $D_{\lambda}^{n, \gamma}$ as follows:

$$
\begin{aligned}
D^{0} f(z)= & f(z), \\
D_{\lambda}^{1, \gamma} f(z)= & (1-\lambda) \Omega^{\gamma} f(z)+\lambda z\left(\Omega^{\gamma} f(z)\right)^{\prime} \\
= & D_{\lambda}^{\gamma}(f(z)), \quad \lambda \geq 0,0 \leq \gamma<1, \\
D_{\lambda}^{2, \gamma} f(z)= & D_{\lambda}^{\gamma}\left(D_{\lambda}^{1, \gamma} f(z)\right), \\
& \vdots \\
D_{\lambda}^{n, \gamma} f(z)= & D_{\lambda}^{\gamma}\left(D_{\lambda}^{n-1, \gamma} f(z)\right), \quad n \in \mathbb{N} .
\end{aligned}
$$

If $f$ is given by (1.1), then by (1.3), (1.4) and (1.5), we see that

$$
D_{\lambda}^{n, \gamma} f(z)=z+\sum_{k=2}^{\infty} \Psi_{k, n}(\gamma, \lambda) a_{k} z^{k}, \quad n \in \mathbb{N}_{0}=\mathbb{N} \cup\{0\},
$$

where

$$
\Psi_{k, n}(\gamma, \lambda)=\left[\frac{\Gamma(k+1) \Gamma(2-\gamma)}{\Gamma(k+1-\gamma)}(1+(k-1) \lambda)\right]^{n} .
$$

Remark 1.1. (i) When $\gamma=0$, we get Al-Oboudi differential operator [2].

(ii) When $\gamma=0$ and $\lambda=1$, we get Sălăgean differential operator [14].

(iii) When $n=1$ and $\lambda=0$, we get Owa-Srivastava fractional differential operator [10].

Let us define the classes $\mathcal{S}_{\gamma, \lambda}^{n}(\beta, b)$ and $\mathcal{K}_{\gamma, \lambda}^{n}(\beta, b)$.

Let $\mathcal{S}_{\gamma, \lambda}^{n}(\beta, b)$ be the class of functions $f \in \mathcal{A}$ satisfying

$$
\Re\left\{1+\frac{1}{b}\left(\frac{z\left(D_{\lambda}^{n, \gamma} f(z)\right)^{\prime}}{D_{\lambda}^{n, \gamma} f(z)}-1\right)\right\}>\beta
$$

for all $z \in \mathbb{U}$, where $b \in \mathbb{C}-\{0\}$ and $0 \leq \beta<1$. 
Let $\mathcal{K}_{\gamma, \lambda}^{n}(\beta, b)$ be the class of functions $f \in \mathcal{A}$ satisfying

$$
\Re\left\{1+\frac{1}{b} \frac{z\left(D_{\lambda}^{n, \gamma} f(z)\right)^{\prime \prime}}{\left(D_{\lambda}^{n, \gamma} f(z)\right)^{\prime}}\right\}>\beta
$$

for all $z \in \mathbb{U}$, where $b \in \mathbb{C}-\{0\}$ and $0 \leq \beta<1$.

We note that $f \in \mathcal{K}_{\gamma, \lambda}^{n}(\beta, b)$ if and only if $z f^{\prime} \in \mathcal{S}_{\gamma, \lambda}^{n}(\beta, b)$.

Remark 1.2. We have the classes

(i) $\mathcal{S}_{\gamma, \lambda}^{0}(\beta, b) \equiv \mathcal{S}_{0,0}^{1}(\beta, b) \equiv \mathcal{S}_{\beta}^{*}(b)$ and $\mathcal{K}_{\gamma, \lambda}^{0}(\beta, b) \equiv \mathcal{K}_{0,0}^{1}(\beta, b) \equiv \mathcal{C}_{\beta}(b)$ defined by Frasin $[6]$.

(ii) $\mathcal{S}_{\gamma, \lambda}^{0}(\beta, 1) \equiv \mathcal{S}_{0,0}^{1}(\beta, 1) \equiv \mathcal{S}^{*}(\beta)$ and $\mathcal{K}_{\gamma, \lambda}^{0}(\beta, 1) \equiv \mathcal{K}_{0,0}^{1}(\beta, 1) \equiv \mathcal{K}(\beta)$ which are the classes of starlike functions of order $\beta$ and convex functions of order $\beta$ in $\mathbb{U}$, respectively.

(iii) $\mathcal{S}_{\gamma, \lambda}^{0}(0,1) \equiv \mathcal{S}_{0,0}^{1}(0,1) \equiv \mathcal{S}^{*}$ and $\mathcal{K}_{\gamma, \lambda}^{0}(0,1) \equiv \mathcal{K}_{0,0}^{1}(0,1) \equiv \mathcal{K}$ which are familiar classes of starlike and convex functions in $\mathbb{U}$, respectively.

(iv) $\mathcal{S}_{0,1}^{n}(\beta, 1) \equiv \mathcal{S}_{n}(\beta)$ which is the class of $n$-starlike functions of order $\beta$ defined by Sălăgean [14].

Observe that if $f \in \mathcal{S}_{\gamma, \lambda}^{n}(\beta, b)\left(\right.$ or $\left.\mathcal{K}_{\gamma, \lambda}^{n}(\beta, b)\right)$, then $D_{\lambda}^{n, \gamma} f \in \mathcal{S}_{\beta}^{*}(b)\left(\right.$ or $\left.\mathcal{C}_{\beta}(b)\right)$.

Now we define new classes by means of the generalized Al-Oboudi differential operator $D_{\lambda}^{n, \gamma}$ as follows:

A function $f \in \mathcal{A}$ is in the class $\mathcal{S D}_{\gamma, \lambda}^{n}(\alpha, \beta, b)$ if

$$
\Re\left\{1+\frac{1}{b}\left(\frac{z\left(D_{\lambda}^{n, \gamma} f(z)\right)^{\prime}}{D_{\lambda}^{n, \gamma} f(z)}-1\right)\right\}>\alpha\left|\frac{1}{b}\left(\frac{z\left(D_{\lambda}^{n, \gamma} f(z)\right)^{\prime}}{D_{\lambda}^{n, \gamma} f(z)}-1\right)\right|+\beta \quad(z \in \mathbb{U})
$$

where $\alpha \geq 0, \beta \in[-1,1), \alpha+\beta \geq 0$ and $b \in \mathbb{C}-\{0\}$.

A function $f \in \mathcal{A}$ is in the class $\mathcal{K D}_{\gamma, \lambda}^{n}(\alpha, \beta, b)$ if

$$
\Re\left\{1+\frac{1}{b} \frac{z\left(D_{\lambda}^{n, \gamma} f(z)\right)^{\prime \prime}}{\left(D_{\lambda}^{n, \gamma} f(z)\right)^{\prime}}\right\}>\alpha\left|\frac{1}{b} \frac{z\left(D_{\lambda}^{n, \gamma} f(z)\right)^{\prime \prime}}{\left(D_{\lambda}^{n, \gamma} f(z)\right)^{\prime}}\right|+\beta \quad(z \in \mathbb{U})
$$

where $\alpha \geq 0, \beta \in[-1,1), \alpha+\beta \geq 0$ and $b \in \mathbb{C}-\{0\}$.

We note that $f \in \mathcal{K D}_{\gamma, \lambda}^{n}(\alpha, \beta, b)$ if and only if $z f^{\prime} \in \mathcal{S D}_{\gamma, \lambda}^{n}(\alpha, \beta, b)$.

Geometric interpretation. $f \in \mathcal{S D}_{\gamma, \lambda}^{n}(\alpha, \beta, b)$ and $f \in \mathcal{K} \mathcal{D}_{\gamma, \lambda}^{n}(\alpha, \beta, b)$ if and only if $1+\frac{1}{b}\left(\frac{z\left(D_{\lambda}^{n, \gamma} f(z)\right)^{\prime}}{D_{\lambda}^{n, \gamma} f(z)}-1\right)$ and $1+\frac{1}{b} \frac{z\left(D_{\lambda}^{n, \gamma} f(z)\right)^{\prime \prime}}{\left(D_{\lambda}^{n, \gamma} f(z)\right)^{\prime}}$, respectively, take all values in the conic domain $R_{\alpha, \beta}$ which is included in the right half plane such that

$$
R_{\alpha, \beta}=\left\{u+i v: u>\alpha \sqrt{(u-1)^{2}+v^{2}}+\beta\right\} .
$$


From elementary computations we see that $\partial R_{\alpha, \beta}$,

$$
\partial R_{\alpha, \beta}=\left\{u+i v: u^{2}=\left(\alpha \sqrt{(u-1)^{2}+v^{2}}+\beta\right)^{2}\right\},
$$

represents the conic sections symmetric about the real axis. Thus $R_{\alpha, \beta}$ is an elliptic domain for $\alpha>1$, a parabolic domain for $\alpha=1$, a hyperbolic domain for $0<\alpha<1$ and a right half plane $u>\beta$ for $\alpha=0$.

By virtue of (1.6), (1.7) and the properties of the domain $R_{\alpha, \beta}$, we have, respectively

$$
\Re\left\{1+\frac{1}{b}\left(\frac{z\left(D_{\lambda}^{n, \gamma} f(z)\right)^{\prime}}{D_{\lambda}^{n, \gamma} f(z)}-1\right)\right\}>\frac{\alpha+\beta}{\alpha+1}
$$

and

$$
\Re\left\{1+\frac{1}{b} \frac{z\left(D_{\lambda}^{n, \gamma} f(z)\right)^{\prime \prime}}{\left(D_{\lambda}^{n, \gamma} f(z)\right)^{\prime}}\right\}>\frac{\alpha+\beta}{\alpha+1},
$$

which means that

$$
f \in \mathcal{S D}_{\gamma, \lambda}^{n}(\alpha, \beta, b) \Rightarrow D_{\lambda}^{n, \gamma} f \in \mathcal{S}_{\gamma, \lambda}^{n}\left(\frac{\alpha+\beta}{\alpha+1}, b\right)
$$

and

$$
f \in \mathcal{K D}_{\gamma, \lambda}^{n}(\alpha, \beta, b) \Rightarrow D_{\lambda}^{n, \gamma} f \in \mathcal{K}_{\gamma, \lambda}^{n}\left(\frac{\alpha+\beta}{\alpha+1}, b\right)
$$

Remark 1.3. We have the classes

(i) $\mathcal{S D}_{\gamma, \lambda}^{n}(0, \beta, b) \equiv \mathcal{S}_{\gamma, \lambda}^{n}(\beta, b)$ and $\mathcal{K D}_{\gamma, \lambda}^{n}(0, \beta, b) \equiv \mathcal{K}_{\gamma, \lambda}^{n}(\beta, b)$.

(ii) $\mathcal{S D}_{\gamma, \lambda}^{n}(\alpha, \beta, 1) \equiv \mathcal{S P}_{\gamma, \lambda}^{n}(\alpha, \beta)$ and $\mathcal{K D}_{\gamma, \lambda}^{n}(\alpha, \beta, 1) \equiv \mathcal{U C V}_{\gamma, \lambda}^{n}(\alpha, \beta)$ (AlOboudi and Al-Amoudi [3]).

(iii) $\mathcal{S} \mathcal{D}_{\gamma, \lambda}^{0}(\alpha, \beta, 1) \equiv \mathcal{S} \mathcal{D}_{0,0}^{1}(\alpha, \beta, 1) \equiv \mathcal{S D}(\alpha, \beta)$ and $\mathcal{K D}_{\gamma, \lambda}^{0}(\alpha, \beta, 1) \equiv \mathcal{K D}_{0,0}^{1}(\alpha, \beta, 1) \equiv \mathcal{K D}(\alpha, \beta)$ (Shams et al. [15]).

(iv) $\mathcal{S D}_{0,1}^{n}(\alpha, \beta, 1) \equiv \mathcal{U S}_{n}(\alpha, \beta)$ which is the class of $n$-uniform starlike functions of order $\beta$ and type $\alpha$ (Acu and Owa [1]).

$(v) \mathcal{S D}_{\gamma, \lambda}^{0}(0, \beta, b) \equiv \mathcal{S D}_{0,0}^{1}(0, \beta, b) \equiv \mathcal{S}_{\beta}^{*}(b)$ and $\mathcal{K D}_{\gamma, \lambda}^{0}(0, \beta, b) \equiv \mathcal{K D}_{0,0}^{1}(0, \beta, b) \equiv$ $\mathcal{S} \mathcal{D}_{0,1}^{1}(0, \beta, b) \equiv \mathcal{C}_{\beta}(b)($ Frasin $[6])$.

$(v i) S \mathcal{D}_{\gamma, \lambda}^{0}(0, \beta, 1) \equiv \mathcal{S} \mathcal{D}_{0,0}^{1}(0, \beta, 1) \equiv \mathcal{S}^{*}(\beta)$ and $\mathcal{K} \mathcal{D}_{\gamma, \lambda}^{0}(0, \beta, 1) \equiv \mathcal{K D}_{0,0}^{1}(0, \beta, 1) \equiv$ $\mathcal{S D}_{0,1}^{1}(0, \beta, 1) \equiv \mathcal{K}(\beta)$.

(vii) $\mathcal{S D}_{\gamma, \lambda}^{0}(0,0,1) \equiv \mathcal{S} \mathcal{D}_{0,0}^{1}(0,0,1) \equiv \mathcal{S}^{*}$ and $\mathcal{K D}_{\gamma, \lambda}^{0}(0,0,1) \equiv \mathcal{K D}_{0,0}^{1}(0,0,1) \equiv$ $\mathcal{S D}_{0,1}^{1}(0,0,1) \equiv \mathcal{K}$.

(viii) $\mathcal{S D}_{\gamma, \lambda}^{0}(\alpha, \beta, 1) \equiv \mathcal{S D}_{0,0}^{1}(\alpha, \beta, 1) \equiv \mathcal{S P}(\alpha, \beta)$ and $\mathcal{K D}_{\gamma, \lambda}^{0}(\alpha, \beta, 1) \equiv$ $\mathcal{K D}_{0,0}^{1}(\alpha, \beta, 1) \equiv \mathcal{S D}_{0,1}^{1}(\alpha, \beta, 1) \equiv \mathcal{U} \mathcal{U} \mathcal{V}(\alpha, \beta)$ which are uniformly starlike and convex functions, respectively, of order $\beta$ and type $\alpha$ (Bharati et al. [4]). 
$(i x) \mathcal{S D}_{\gamma, \lambda}^{0}(1, \beta, 1) \equiv \mathcal{S} \mathcal{D}_{0,0}^{1}(1, \beta, 1) \equiv \mathcal{S P}(\beta)$ and $\mathcal{K D}_{\gamma, \lambda}^{0}(1, \beta, 1) \equiv \mathcal{K} \mathcal{D}_{0,0}^{1}(1, \beta, 1) \equiv$ $\mathcal{S D}_{0,1}^{1}(1, \beta, 1) \equiv \mathcal{U} \mathcal{C V}(\beta)(\mathrm{R} \varnothing$ nning $[12])$.

$(x) \mathcal{S D}_{\gamma, \lambda}^{0}(1,0,1) \equiv \mathcal{S} \mathcal{D}_{0,0}^{1}(1,0,1) \equiv \mathcal{S P}(\mathrm{R} ø$ nning $[13])$ and $\mathcal{K D}_{\gamma, \lambda}^{0}(1,0,1) \equiv$ $\mathcal{K D}_{0,0}^{1}(1,0,1) \equiv \mathcal{S} \mathcal{D}_{0,1}^{1}(1,0,1) \equiv \mathcal{U C V}$ which is the class of uniformly convex functions (Goodman $[7]$ ).

(xi) $\mathcal{S D}_{\gamma, 0}^{1}(0, \beta, 1) \equiv \mathcal{S T}_{\gamma}(\beta)$ (Srivastava et al. [16]).

(xii) $\mathcal{S D}_{\gamma, 0}^{1}(1,0,1) \equiv \mathcal{S P}_{\gamma}$ (Srivastava and Mishra [17]).

Observe that if $f \in \mathcal{S D}_{\gamma, \lambda}^{n}(\alpha, \beta, b)\left(\right.$ or $\left.\mathcal{K} \mathcal{D}_{\gamma, \lambda}^{n}(\alpha, \beta, b)\right)$, then $D_{\lambda}^{n, \gamma} f \in \mathcal{S} \mathcal{D}(\alpha, \beta, b)$ (or $\mathcal{K D}(\alpha, \beta, b)$ ).

For the classes $\mathcal{S} \mathcal{D}(\alpha, \beta)$ and $\mathcal{K} \mathcal{D}(\alpha, \beta)$, Shams et al. [15] have shown some sufficient conditions for $f$ to be in the classes $\mathcal{S} \mathcal{D}(\alpha, \beta)$ and $\mathcal{K} \mathcal{D}(\alpha, \beta)$.

In [9], Owa et al. have investigated coefficient inequalities for $f$ belonging to the classes $\mathcal{S} \mathcal{D}(\alpha, \beta)$ and $\mathcal{K} \mathcal{D}(\alpha, \beta)$.

The purpose of this paper is to generalize the results of [9] using generalized Al-Oboudi differential operator.

\section{Main results}

Theorem 2.1. If $f \in \mathcal{S D}_{\gamma, \lambda}^{n}(\alpha, \beta, b)$ with $0 \leq \alpha \leq \beta$, then $f \in \mathcal{S}_{\gamma, \lambda}^{n}(\delta, b)$ where $\delta=\frac{\beta-\alpha}{1-\alpha}$.

Proof. Let $f \in \mathcal{S D}_{\gamma, \lambda}^{n}(\alpha, \beta, b)$. Then we have

$$
\Re\left\{1+\frac{1}{b}\left(\frac{z\left(D_{\lambda}^{n, \gamma} f(z)\right)^{\prime}}{D_{\lambda}^{n, \gamma} f(z)}-1\right)\right\}>\alpha \Re\left\{\frac{1}{b}\left(\frac{z\left(D_{\lambda}^{n, \gamma} f(z)\right)^{\prime}}{D_{\lambda}^{n, \gamma} f(z)}-1\right)\right\}+\beta
$$

or equivalently

$$
(1-\alpha) \Re\left\{1+\frac{1}{b}\left(\frac{z\left(D_{\lambda}^{n, \gamma} f(z)\right)^{\prime}}{D_{\lambda}^{n, \gamma} f(z)}-1\right)\right\}>\beta-\alpha \quad(z \in \mathbb{U}) .
$$

If $0 \leq \alpha \leq \beta$, then we get

$$
0 \leq \frac{\beta-\alpha}{1-\alpha}<1
$$

Corollary 2.2. If $f \in \mathcal{K D}_{\gamma, \lambda}^{n}(\alpha, \beta, b)$ with $0 \leq \alpha \leq \beta$, then $f \in \mathcal{K}_{\gamma, \lambda}^{n}(\delta, b)$ where $\delta=\frac{\beta-\alpha}{1-\alpha}$.

Theorem 2.3. If $f \in S \mathcal{D}_{\gamma, \lambda}^{n}(\alpha, \beta, b)$ with $0 \leq \alpha \leq \beta$, then

$$
\left|a_{2}\right| \leq \frac{2|b|(1-\beta)}{\Psi_{2, n}(\gamma, \lambda)(1-\alpha)}
$$


and

$$
\left|a_{k}\right| \leq \frac{2|b|(1-\beta)}{\Psi_{k, n}(\gamma, \lambda)(k-1)(1-\alpha)} \prod_{j=1}^{k-2}\left(1+\frac{2|b|(1-\beta)}{j(1-\alpha)}\right) \quad(k \geq 3) .
$$

Proof. We note that for $f \in \mathcal{S} \mathcal{D}_{\gamma, \lambda}^{n}(\alpha, \beta, b)$ with $0 \leq \alpha \leq \beta$,

$$
\Re\left\{1+\frac{1}{b}\left(\frac{z\left(D_{\lambda}^{n, \gamma} f(z)\right)^{\prime}}{D_{\lambda}^{n, \gamma} f(z)}-1\right)\right\}>\frac{\beta-\alpha}{1-\alpha} \quad(z \in \mathbb{U}) .
$$

Let us define the function $p(z)$ by

$$
p(z)=\frac{(1-\alpha)\left[1+\frac{1}{b}\left(\frac{z\left(D_{\lambda}^{n, \gamma} f(z)\right)^{\prime}}{D_{\lambda}^{n, \gamma} f(z)}-1\right)\right]-(\beta-\alpha)}{1-\beta} \quad(z \in \mathbb{U}) .
$$

Hence $p(z)$ is analytic in $\mathbb{U}$ with $p(0)=1$ and $\Re\{p(z)\}>0(z \in \mathbb{U})$. Let

$$
p(z)=1+p_{1} z+p_{2} z^{2}+\cdots .
$$

So we obtain

$$
1+\frac{1}{b}\left(\frac{z\left(D_{\lambda}^{n, \gamma} f(z)\right)^{\prime}}{D_{\lambda}^{n, \gamma} f(z)}-1\right)=1+\frac{1-\beta}{1-\alpha}\left(p_{1} z+p_{2} z^{2}+\cdots\right)
$$

or equivalently

$$
z\left(D_{\lambda}^{n, \gamma} f(z)\right)^{\prime}-D_{\lambda}^{n, \gamma} f(z)=b \frac{1-\beta}{1-\alpha}\left(D_{\lambda}^{n, \gamma} f(z)\right)\left(p_{1} z+p_{2} z^{2}+\cdots\right) .
$$

The last equality implies that

$$
\begin{gathered}
\Psi_{k, n}(\gamma, \lambda)(k-1) a_{k}=\frac{b(1-\beta)}{1-\alpha}\left\{p_{k-1}+\Psi_{2, n}(\gamma, \lambda) a_{2} p_{k-2}+\Psi_{3, n}(\gamma, \lambda) a_{3} p_{k-3}\right. \\
\left.+\cdots+\Psi_{k-1, n}(\gamma, \lambda) a_{k-1} p_{1}\right\} .
\end{gathered}
$$

Applying the coefficient estimates $\left|p_{k}\right| \leq 2(k \geq 1)$ for Carathéodory functions [5], we get

$$
\begin{aligned}
\left|a_{k}\right| \leq \frac{2|b|(1-\beta)}{\Psi_{k, n}(\gamma, \lambda)(k-1)(1-\alpha)} & \left\{1+\Psi_{2, n}(\gamma, \lambda)\left|a_{2}\right|\right. \\
+ & \Psi_{3, n}(\gamma, \lambda)\left|a_{3}\right| \\
+ & \left.\cdots+\Psi_{k-1, n}(\gamma, \lambda)\left|a_{n-1}\right|\right\} .
\end{aligned}
$$

For $k=2$,

$$
\left|a_{2}\right| \leq \frac{2|b|(1-\beta)}{\Psi_{2, n}(\gamma, \lambda)(1-\alpha)},
$$


which proves (2.1).

For $k=3$,

$$
\left|a_{3}\right| \leq \frac{2|b|(1-\beta)}{\Psi_{3, n}(\gamma, \lambda) 2(1-\alpha)}\left(1+\frac{2|b|(1-\beta)}{1-\alpha}\right) .
$$

Therefore (2.2) holds for $k=3$.

Assume that (2.3) is true for $k=m$. Then we obtain

$$
\begin{aligned}
\left|a_{m+1}\right| \leq & \frac{2|b|(1-\beta)}{\Psi_{m+1, n}(\gamma, \lambda) m(1-\alpha)}\left\{1+\frac{2|b|(1-\beta)}{1-\alpha}\right. \\
& +\frac{2|b|(1-\beta)}{2(1-\alpha)}\left(1+\frac{2|b|(1-\beta)}{1-\alpha}\right) \\
& +\cdots+\frac{2|b|(1-\beta)}{(m-1)(1-\alpha)} \prod_{j=1}^{m-2}\left(1+\frac{2|b|(1-\beta)}{j(1-\alpha)}\right) \\
= & \frac{2|b|(1-\beta)}{\Psi_{m+1, n}(\gamma, \lambda) m(1-\alpha)} \prod_{j=1}^{m-1}\left(1+\frac{2|b|(1-\beta)}{j(1-\alpha)}\right) .
\end{aligned}
$$

So $(2.2)$ is true for $k=m+1$.

Consequently, using mathematical induction, we have proved that (2.2) holds true for all $k \geq 3$.

Corollary 2.4. Setting $\alpha=0$ in Theorem 2.3, we have

$$
\left|a_{k}\right| \leq \frac{\prod_{j=0}^{k-2}(j+2|b|(1-\beta))}{\Psi_{k, n}(\gamma, \lambda)(k-1) !} \quad(k \geq 2) .
$$

Corollary 2.5. If we set $n=0,|b|=1$ or $n=1, \gamma=\lambda=0,|b|=1$ in Corollary 2.4, then we have

$$
\left|a_{k}\right| \leq \frac{\prod_{j=2}^{k}(j-2 \beta)}{(k-1) !} \quad(k \geq 2)
$$

given by Robertson [11].

Theorem 2.6.If $f \in \mathcal{K} \mathcal{D}_{\gamma, \lambda}^{n}(\alpha, \beta, b)$ with $0 \leq \alpha \leq \beta$, then

$$
\left|a_{2}\right| \leq \frac{|b|(1-\beta)}{\Psi_{2, n}(\gamma, \lambda)(1-\alpha)}
$$

and

$$
\left|a_{k}\right| \leq \frac{2|b|(1-\beta)}{\Psi_{k, n}(\gamma, \lambda) k(k-1)(1-\alpha)} \prod_{j=1}^{k-2}\left(1+\frac{2|b|(1-\beta)}{j(1-\alpha)}\right) \quad(k \geq 3) .
$$


Corollary 2.7. Setting $\alpha=0$ in Theorem 2.6, we have

$$
\left|a_{k}\right| \leq \frac{\prod_{j=0}^{k-2}(j+2|b|(1-\beta))}{\Psi_{k, n}(\gamma, \lambda) k !} \quad(k \geq 2) .
$$

Corollary 2.8. If we set $n=0,|b|=1$ or $n=1, \gamma=\lambda=0,|b|=1$ in Corollary 2.7, then we have

$$
\left|a_{k}\right| \leq \frac{\prod_{j=2}^{k}(j-2 \beta)}{k !} \quad(k \geq 2)
$$

given by Robertson [11].

Theorem 2.9. If $f \in S \mathcal{D}_{\gamma, \lambda}^{n}(\alpha, \beta, b)$ with $0 \leq \alpha \leq \beta$, then

$$
\begin{aligned}
& \max \left\{0,|z|-\frac{2|b|(1-\beta)}{\Psi_{2, n}(\gamma, \lambda)(1-\alpha)}|z|^{2}\right. \\
& \left.-\sum_{k=3}^{\infty} \frac{2|b|(1-\beta)}{\Psi_{k, n}(\gamma, \lambda)(k-1)(1-\alpha)}\left(\prod_{j=1}^{k-2}\left(1+\frac{2|b|(1-\beta)}{j(1-\alpha)}\right)\right)|z|^{k}\right\} \\
\leq & |f(z)| \leq|z|+\frac{2|b|(1-\beta)}{\Psi_{2, n}(\gamma, \lambda)(1-\alpha)}|z|^{2} \\
& +\sum_{k=3}^{\infty} \frac{2|b|(1-\beta)}{\Psi_{k, n}(\gamma, \lambda)(k-1)(1-\alpha)}\left(\prod_{j=1}^{k-2}\left(1+\frac{2|b|(1-\beta)}{j(1-\alpha)}\right)\right)|z|^{k}
\end{aligned}
$$

and

$$
\begin{aligned}
& \quad \max \left\{0,1-\frac{4|b|(1-\beta)}{\Psi_{2, n}(\gamma, \lambda)(1-\alpha)}|z|\right. \\
& \left.\quad-\sum_{k=3}^{\infty} \frac{2 k|b|(1-\beta)}{\Psi_{k, n}(\gamma, \lambda)(k-1)(1-\alpha)}\left(\prod_{j=1}^{k-2}\left(1+\frac{2|b|(1-\beta)}{j(1-\alpha)}\right)\right)|z|^{k-1}\right\} \\
& \leq \quad\left|f^{\prime}(z)\right| \leq 1+\frac{4|b|(1-\beta)}{\Psi_{2, n}(\gamma, \lambda)(1-\alpha)}|z| \\
& \quad+\sum_{k=3}^{\infty} \frac{2 k|b|(1-\beta)}{\Psi_{k, n}(\gamma, \lambda)(k-1)(1-\alpha)}\left(\prod_{j=1}^{k-2}\left(1+\frac{2|b|(1-\beta)}{j(1-\alpha)}\right)\right)|z|^{k-1} .
\end{aligned}
$$


Theorem 2.10. If $f \in \mathcal{K} \mathcal{D}_{\gamma, \lambda}^{n}(\alpha, \beta, b)$ with $0 \leq \alpha \leq \beta$, then

$$
\begin{aligned}
& \max \left\{0,|z|-\frac{|b|(1-\beta)}{\Psi_{2, n}(\gamma, \lambda)(1-\alpha)}|z|^{2}\right. \\
& \left.-\sum_{k=3}^{\infty} \frac{2|b|(1-\beta)}{\Psi_{k, n}(\gamma, \lambda) k(k-1)(1-\alpha)}\left(\prod_{j=1}^{k-2}\left(1+\frac{2|b|(1-\beta)}{j(1-\alpha)}\right)\right)|z|^{k}\right\} \\
\leq & |f(z)| \leq|z|+\frac{|b|(1-\beta)}{\Psi_{2, n}(\gamma, \lambda)(1-\alpha)}|z|^{2} \\
& +\sum_{k=3}^{\infty} \frac{2|b|(1-\beta)}{\Psi_{k, n}(\gamma, \lambda) k(k-1)(1-\alpha)}\left(\prod_{j=1}^{k-2}\left(1+\frac{2|b|(1-\beta)}{j(1-\alpha)}\right)\right)|z|^{k}
\end{aligned}
$$

and

$$
\begin{aligned}
& \max \left\{0,1-\frac{2|b|(1-\beta)}{\Psi_{2, n}(\gamma, \lambda)(1-\alpha)}|z|\right. \\
& \left.-\sum_{k=3}^{\infty} \frac{2|b|(1-\beta)}{\Psi_{k, n}(\gamma, \lambda)(k-1)(1-\alpha)}\left(\prod_{j=1}^{k-2}\left(1+\frac{2|b|(1-\beta)}{j(1-\alpha)}\right)\right)|z|^{k-1}\right\} \\
\leq & \left|f^{\prime}(z)\right| \leq 1+\frac{2|b|(1-\beta)}{\Psi_{2, n}(\gamma, \lambda)(1-\alpha)}|z| \\
& +\sum_{k=3}^{\infty} \frac{2|b|(1-\beta)}{\Psi_{k, n}(\gamma, \lambda)(k-1)(1-\alpha)}\left(\prod_{j=1}^{k-2}\left(1+\frac{2|b|(1-\beta)}{j(1-\alpha)}\right)\right)|z|^{k-1} .
\end{aligned}
$$

\section{References}

[1] M. Acu and S. Owa, Convex functions associated with some hyperbola, J. Approx. Theory Appl., 1(2005), 37-40.

[2] F. M. Al-Oboudi, On univalent functions defined by a generalized Sălăgean operator, Int. J. Math. Math. Sci. 2004, no. 25-28, 1429-1436.

[3] F. M. Al-Oboudi and K. A. Al-Amoudi, On classes of analytic functions related to conic domains, J. Math. Anal. Appl., 339(2008), 655-667.

[4] R. Bharati, R. Parvatham and A. Swaminathan, On subclasses of uniformly convex functions and corresponding class of starlike functions, Tamkang J. Math., 28(1997), 17-32.

[5] C. Carathéodory, Über den variabilitätsbereich der Fourier'schen konstanten von possitiven harmonischen funktionen, Rend. Circ. Palermo, 32(1911), 193-217. 
[6] B. A. Frasin, Family of analytic functions of complex order, Acta Math. Acad. Paedagog. Nyházi. (N.S.), 22(2006), 179-191.

[7] A. W. Goodman, On uniformly convex functions, Ann. Polon. Math., 56(1991), 87-92.

[8] S. Owa, On the distortion theorems. I, Kyungpook Math. J. 18(1978), 53-59.

[9] S. Owa, Y. Polatoğlu and E. Yavuz, Coefficient inequalities for classes of uniformly starlike and convex functions, J. Inequal. Pure Appl. Math., 7(2006), Article 160, 5 pp.

[10] S. Owa and H. M. Srivastava, Univalent and starlike generalized hypergeometric functions, Canad. J. Math., 39(1987), 1057-1077.

[11] M. S. Robertson, On the theory of univalent functions, Ann. of Math., 37(2)(1936), 374-408.

[12] F. Rønning, On starlike functions associated with parabolic regions, Ann. Univ. Mariae Curie-Sklodowska Sect. A, 45(1991), 117-122.

[13] F. Rønning, Uniformly convex functions and a corresponding class of starlike functions, Proc. Amer. Math. Soc. ,118(1993), 189-196.

[14] G. Ş. Sălăgean, Subclasses of univalent functions, Complex Analysis-Fifth RomanianFinnish seminar, Part 1 (Bucharest, 1981), Lecture Notes in Math., vol. 1013, Springer, Berlin, 1983, pp. 362-372.

[15] S. Shams, S. R. Kulkarni and J. M. Jahangiri, Classes of uniformly starlike and convex functions, Int. J. Math. Math. Sci., 55(2004), 2959-2961.

[16] H. M. Srivastava, A. K. Mishra and M. K. Das, A nested class of analytic functions defined by fractional calculus, Commun. Appl. Anal., 2(1998), 321-332.

[17] H. M. Srivastava and A. K. Mishra, Applications of fractional calculus to parabolic starlike and uniformly convex functions, Comput. Math. Appl., 39(2000), 57-69.

[18] H. M. Srivastava and S. Owa, (Eds.), Univalent Functions, Fractional Calculus, and Their Applications, Ellis Horwood Series: Mathematics and Its Applications, Ellis Horwood, Chichester, UK; JohnWiley \& Sons, New York, NY, USA, 1989. 